\title{
COMPETÊNCIAS GERENCIAIS RELACIONADAS À SEGURANÇA DO PACIENTE: UMA REVISÃO INTEGRATIVA
}

\author{
Management competences related to patient safety: an integrating review
}

\author{
Competencias gerenciales relacionadas a la seguridad del paciente: una revisión integrativa
}

Taís Couto Rego da Paixão ${ }^{1}$, Alexandre Pazetto Balsanelli², Elena Bohomol ${ }^{3}$, Vanessa Ribeiro Neves ${ }^{4}$

RESUMO: Objetivo: Identificar as competências gerenciais relacionadas à segurança do paciente. Método: Trata-se de uma revisão integrativa da literatura. A busca foi realizada em 2017, nos idiomas inglês, português e espanhol, na base de dados Public Medline (PubMed). Os textos incluídos deveriam estar disponíveis na íntegra e abordar a temática proposta. Resultados: Foram selecionados 34 artigos e identificadas 11 competências: liderança, cultura da segurança, trabalho em equipe, comunicação, advocacia, manejo de risco, competência conceitual, competência funcional, inteligência emocional, tomada de decisão e gestão e planejamento centrado na segurança. A identificação das competências possibilitou defini-las no contexto da gestão, quando aplicáveis na área cirúrgica, além de verificar as estratégias para o seu desenvolvimento. Conclusão: Embora tenha sido utilizada apenas uma base de dados, recomenda-se que estudos subsequentes apliquem essas estratégias, o que permitirá aos gestores das instituições planejar a implementação da cultura de segurança de uma maneira mais efetiva e concreta.

Palavras-chave: Competência profissional. Segurança do paciente. Liderança. Sistema de saúde.

ABSTRACT: Objective: To identify management competences related to patient safety. Method: It is an integrative literature review. The research was carried out in 2017, in English, Portuguese and Spanish, in Public Medline (PubMed) database. The texts included should be available in full and address the theme proposed. Results: A total of 34 articles were selected and 11 competences were identified: leadership, safety culture, teamwork, communication, advocacy, risk management, concept competence, functional competence, emotional intelligence, decision making and safety-focused management and planning. Identifying the competences allowed for their definition in the context of management, when applicable in the surgical area, in addition to verifying strategies for their development. Conclusion: Although only one database was used for the research, it is recommended that subsequent studies adopt these strategies, which will allow for institution managers to plan and implement the culture of safety in a more effective and concrete way. Keywords: Professional competence. Patient safety. Leadership. Health System.

RESUMEN: Objetivo: Identificar las competencias gerenciales relacionadas con la seguridad del paciente. Método: Se trata de una revisión integrativa de la literatura. La búsqueda se realizó en 2017, en inglés, portugués y español, en la base de datos Public Medline (PubMed). Los textos incluidos deberían estar disponibles en su totalidad y abordar la temática propuesta. Resultados: Se seleccionaron 34 artículos y se identificaron 11 competencias: liderazgo, cultura de la seguridad, trabajo en equipo, comunicación, abogacía, manejo de riesgos, competencia conceptual, competencia funcional, inteligencia emocional, toma de decisión y gestión y planificación centradas en la seguridad. La identificación de las competencias posibilitó definirlas en el contexto de la gestión, cuando aplicables en el área quirúrgica, además de verificar las estrategias para su desarrollo. Conclusión: Aunque se ha utilizado sólo una base de datos, se recomienda que estudios posteriores apliquen esas estrategias, lo que permitirá a los gestores de las instituciones planificar la aplicación de la cultura de seguridad de una manera más efectiva y concreta.

Palabras clave: Competencia profesional. Seguridad del paciente. Liderazgo. Sistema de salud.

'Enfermeira pela Escola Paulista de Enfermagem (EPE) da Universidade Federal de São Paulo (UNIFESP). Mestranda do Programa de Pós-Graduação Stricto Sensu da EPE-UNIFESP - São Paulo (SP), Brasil. ${ }^{2}$ Enfermeiro pela Faculdade de Medicina de São José do Rio Preto. Doutor em Ciências pela EPE-UNIFESP. Professor adjunto do Departamento de Administração em Serviços de Saúde e Enfermagem (DASSE) da EPE-UNIFESP - São Paulo (SP), Brasil.

${ }^{3}$ Enfermeira pela Universidade de Mogi das Cruzes. Livre-docente em Enfermagem na Escola de Enfermagem da Universidade de São Paulo (USP). Professora adjunta do DASSE da EPE-UNIFESP - São Paulo (SP), Brasil.

“Enfermeira pela EPE-UNIFESP. Doutora em Enfermagem pela EPE-UNIFESP. Professora adjunta do DASSE da EPE-UNIFESP - São Paulo (SP), Brasil. E-mail: vanessa.neves@unifesp.br

Rua Napoleão de Barros, 754 - Vila Clementino - CEP: $04021-002$ - São Paulo (SP), Brasil.

Recebido: 07 ago. 2017 - Aprovado: 18 set. 2017

DOI: $10.5327 / Z 1414-4425201700040009$ 


\section{INTRODUÇÃO}

Apesar dos esforços dos profissionais de saúde, a prática assistencial tem se mostrado uma atividade de geração de riscos para quem a recebe ${ }^{1}$. Nessa perspectiva, um estudo retrospectivo, realizado nos Estados Unidos, no Reino Unido e na Austrália, evidenciou que um em cada dez pacientes hospitalizados está susceptível a sofrer algum tipo de erro durante sua internação, mostrando que permanecer hospitalizado tem sido uma das atividades humanas de maior risco à integridade do indivíduo ${ }^{2}$.

Preocupada com essa questão, a Organização Mundial da Saúde (OMS) publicou, em 2004, um Programa de Aliança Mundial para a Segurança do Paciente e propôs ações com objetivo de enfrentar esse problema e melhorar as práticas no cuidado à saúde. Uma delas foi fazer uma chamada para a segurança do paciente, estabelecendo três desafios globais: "Cuidado limpo é cuidado mais seguro" (2005), "Cirurgias seguras salvam vidas" (2008) e "Uso seguro de medicamentos" (2017). O Brasil, por ser um Estado-membro da OMS, aderiu a todos os desafios e vem desenvolvendo trabalhos para melhoria da atenção à saúde ${ }^{1}$.

Contudo, o progresso para redução do risco associado à intervenção e/ou à hospitalização tem sido moroso ${ }^{3}$, pois as taxas e as incidências de erros continuam praticamente as mesmas em relação às apresentadas em estudos precedentes ${ }^{4}$.

Torna-se necessário, aos profissionais da saúde, propor melhorias com vistas à promoção do cuidado seguro nas instituições, em especial nos hospitais e, principalmente, no centro cirúrgico. Assim, a aquisição de competências relacionadas à gestão desses serviços é imprescindível para o alcance desse objetivo.

As competências referem-se ao conhecimento do indivíduo, às suas habilidades técnicas e não técnicas e a uma atitude proativa; e são definidas como um saber agir responsável e reconhecido, que implica mobilizar, integrar e transferir conhecimentos, recursos e habilidades que agreguem valor econômico à organização e valor social ao indivíduo 5 .

A associação entre competências e segurança do paciente tem sido abordada na literatura ${ }^{6-11}$, com especial destaque para a comunicação ${ }^{8,11}$ e para a liderança ${ }^{10}$. No entanto, sabe-se que, no âmbito da gestão da segurança, é primordial conhecer todas as competências necessárias para torná-la efetiva e eficaz, tendo como foco o cuidado oferecido. Por esse motivo, questiona-se: quais são as competências gerenciais relacionadas à segurança do paciente?

Tal resposta poderá fornecer elementos para a educação permanente nas instituições, subsidiar um plano de desenvolvimento individual aos profissionais e avançar no conhecimento a respeito dessa temática.

\section{OBJETIVO}

Identificar as competências gerenciais relacionadas à segurança do paciente.

\section{MÉTODOS}

Trata-se de uma revisão integrativa, elaborada conforme as seguintes etapas:

1. definição do objetivo da revisão integrativa;

2. estabelecimento de critérios de inclusão e exclusão dos artigos (seleção da amostra);

3. definição das informações a serem extraídas dos artigos selecionados;

4. análise dos resultados;

5. discussão e apresentação dos resultados;

6. apresentação da revisão ${ }^{12}$.

Foram incluídos artigos publicados no período entre 2005 e 2017, nos idiomas inglês, português e espanhol, cujos textos estivessem disponíveis na íntegra na base de dados consultada e abordassem a temática proposta. Vale ressaltar que o recorte temporal foi estabelecido mediante a publicação, em 2005, pela OMS, da Aliança Mundial para a Segurança do Paciente, fato que chamou a atenção do mundo e levou ao desenvolvimento de diversas pesquisas sobre o assunto.

Documentos como carta ao editor, resenhas, editoriais e capítulos de livros foram excluídos, assim como pesquisas publicadas por profissionais de outras áreas do conhecimento, que não do campo da saúde.

Para a seleção dos artigos, foi utilizada a base de dados Public Medline (PubMed), e os descritores: professional competence, leadership e patient safety. A utilização simultânea desses três descritores resultou em poucas publicações, portanto optou-se pela seleção dos artigos capturados nas buscas realizadas com as combinações leadership AND patient safety (255) e professional competence AND patient safety (400), totalizando 655 artigos. A seleção inicial ocorreu pela leitura do título e do resumo dessas publicações; após a aplicação dos critérios de inclusão e exclusão, 34 artigos foram escolhidos para integrar este estudo (Figura 1).

A coleta de dados ocorreu durante o mês de junho de 2017. Os artigos foram lidos na íntegra e analisados quanto à pertinência ao assunto e à similaridade entre os textos, o que permitiu sintetizar o conteúdo acerca de cada competência, identificar as relações existentes entre elas e criar categorias 
para apresentação e discussão de tais achados. Além disso, as publicações foram codificadas, utilizando-se a letra "A" seguida de um número cardinal, para facilitar sua apresentação e identificação nos quadros que compuseram este estudo. Também foram extraídos dos artigos instrumentos de avaliação e técnicas para o desenvolvimento de competências.

Por se tratar de uma revisão integrativa, na qual não houve participação ou envolvimento de seres humanos, não foi necessária aprovação prévia de Comitê de Ética em Pesquisa.

\section{RESULTADOS}

Os 34 artigos referentes às competências voltadas à segurança do paciente foram publicados entre 2008 e 2016, sendo $3 \%$ em $2008,15 \%$ em $2009,12 \%$ em $2010,6 \%$ em $2011,15 \%$ em $2012,25 \%$ em 2013, $6 \%$ em $2014,6 \%$ em 2015 e $12 \%$ em 2016 . Não houve publicações nos anos de 2005, 2006, 2007 e 2017.

Foram identificados 14 países de origem das publicações, sendo que os Estados Unidos concentraram 32\% e o Reino Unido, $25 \%$ dos artigos, seguidos do Canadá, com 12\%, Austrália, Nova Zelândia e Suécia, com 5\%, e Coreia do Sul, Dinamarca, Etiópia, Holanda, Israel, Japão, Suíça e Taiwan, com $2 \%$ das pesquisas cada um (Quadro 1). Não houve, no período analisado, artigos brasileiros sobre o tema na base de dados consultada.

Os artigos selecionados abordaram competências relacionadas à segurança do paciente, descreveram ferramentas que auxiliam na construção dessas ferramentas e apresentaram

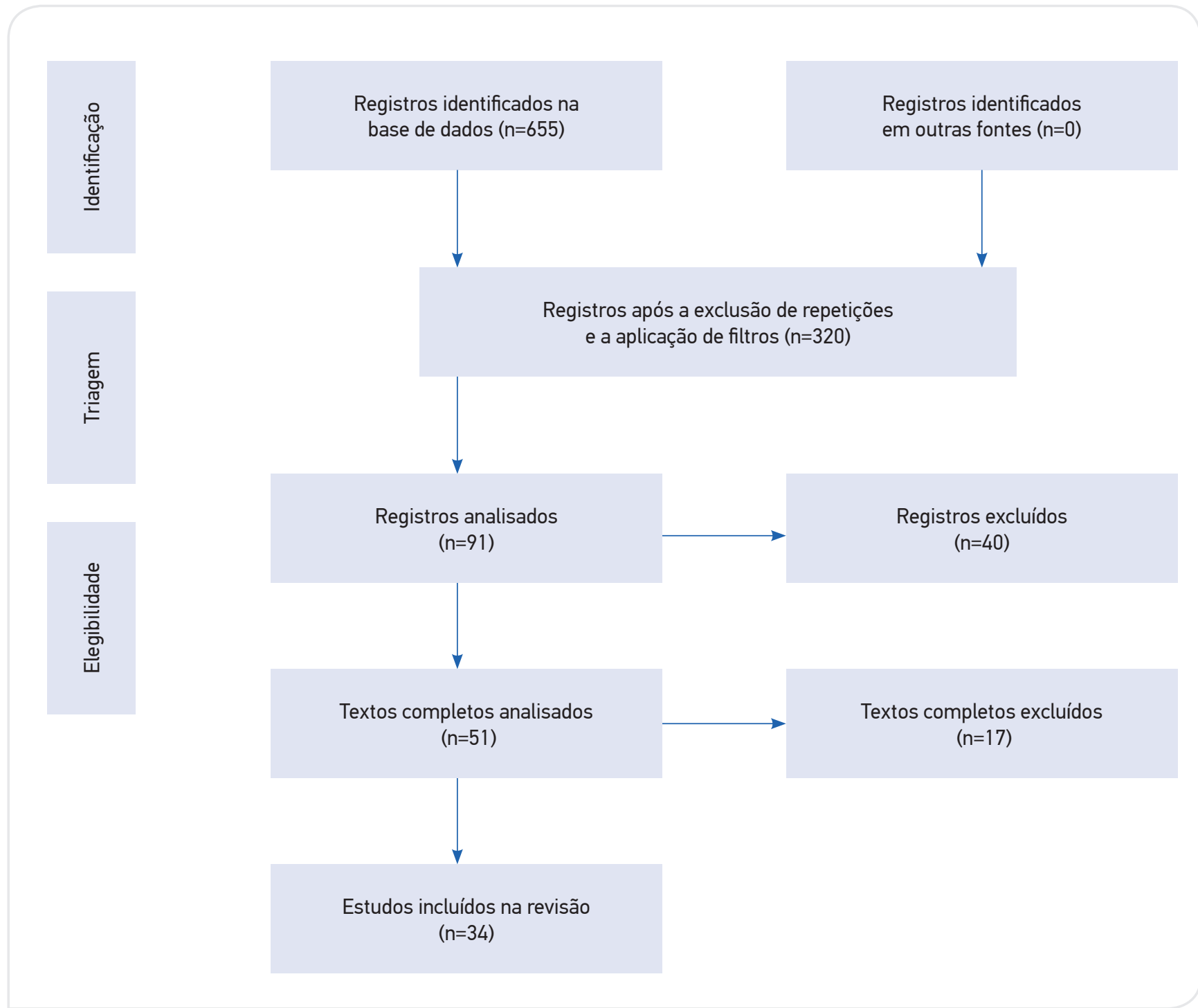

Figura 1. Fluxograma da seleção de publicações. São Paulo, 2017. 
Quadro 1. Distribuição dos artigos selecionados, com demonstração do código, título, ano, país e revista. São Paulo, 2017.

\begin{tabular}{|c|c|c|c|c|}
\hline Código & Título do artigo & Ano & País & Revista \\
\hline $\mathrm{A} 1^{13}$ & $\begin{array}{l}\text { What's makes maternity teams effective and safe? } \\
\text { Lessons from a series of research on teamwork, } \\
\text { leadership and team training }\end{array}$ & 2013 & Reino Unido & $\begin{array}{l}\text { Acta Obstetricia et } \\
\text { Gynecologica Scandinavica }\end{array}$ \\
\hline$A 2^{14}$ & Training and simulation for patient safety & 2010 & $\begin{array}{l}\text { Reino Unido, Etiópia, } \\
\text { Estados Unidos, Japão, } \\
\quad \text { Israel e Canadá }\end{array}$ & Quality \& Safety Health Care \\
\hline$A 3^{15}$ & $\begin{array}{c}\text { The Patient Safety OSCE for PGY-1 Residents: A Centralized } \\
\text { Response to the Challenge of Culture Change }\end{array}$ & 2009 & Estados Unidos & $\begin{array}{l}\text { Teaching and Learning in } \\
\text { Medicine }\end{array}$ \\
\hline$A 4^{16}$ & $\begin{array}{c}\text { The patient safety chain: Transformational leadership's effect } \\
\text { on patient safety culture, initiatives, and outcomes }\end{array}$ & 2009 & Estados Unidos & $\begin{array}{l}\text { Journal of Operations } \\
\text { Management }\end{array}$ \\
\hline$A 5^{6}$ & $\begin{array}{c}\text { The H-PEPSS: an instrument to measure health } \\
\text { professionals' perceptions of patient safety competence at } \\
\text { entry into practice }\end{array}$ & 2012 & Canadá & BMJ Quality \& Safety \\
\hline$A 6^{17}$ & Team training for surgical trainee & 2011 & Estados Unidos & $\begin{array}{l}\text { The Surgeon, Jornal of the } \\
\text { Colleges of Surgeons of } \\
\text { Edinburgh and Ireland }\end{array}$ \\
\hline$A 7^{7}$ & $\begin{array}{l}\text { Self-reported patient safety competence among new } \\
\text { graduates in medicine, nursing and pharmacy }\end{array}$ & 2013 & Canadá & BMJ Quality \& Safety \\
\hline$A 8^{18}$ & $\begin{array}{c}\text { Safety in numbers 2: Competency modelling } \\
\text { and diagnostic error assessment in medication dosage } \\
\text { calculation problem-solving }\end{array}$ & 2013 & $\begin{array}{l}\text { Reino Unido, Nova } \\
\text { Zelândia, Estados } \\
\quad \text { Unidos }\end{array}$ & Nurse Education in Practice \\
\hline A $9^{19}$ & Safe eye surgery: non-technical aspects & 2011 & Reino Unido & Eye \\
\hline $\mathrm{A} 10^{2}$ & $\begin{array}{c}\text { Review article: Simulation: a means to address and improve } \\
\text { patient safety }\end{array}$ & 2013 & Canadá & $\begin{array}{l}\text { Canadian Journal of } \\
\text { Anesthesia }\end{array}$ \\
\hline A $11^{20}$ & $\begin{array}{c}\text { Quality and Safety Initiatives in the Future } \\
\text { Practice of Surgery: Meeting Patient Demands for } \\
\text { Enhanced Professionalism }\end{array}$ & 2009 & Estados Unidos & Surgery Today \\
\hline $\mathrm{A} 12^{21}$ & Patient Safety in the Obstetric and Gynecologic Office Setting & 2013 & Estados Unidos & $\begin{array}{l}\text { Obstetrics \& Gynecology } \\
\text { Clinics of North America }\end{array}$ \\
\hline $\mathrm{A} 13^{8}$ & $\begin{array}{c}\text { Patient safety and communication: A new assessment for } \\
\text { doctors trained in countries where language differs from } \\
\text { that of the host country: Results of a pilot using a domain- } \\
\text { based assessment }\end{array}$ & 2014 & Reino Unido & $\begin{array}{l}\text { Patient Education and } \\
\quad \text { Counseling }\end{array}$ \\
\hline $\mathrm{A} 14^{9}$ & $\begin{array}{l}\text { Nurses values, attitudes and behavior } \\
\text { related to falls prevention }\end{array}$ & 2009 & Austrália & Journal of Clinical Nursing \\
\hline $\mathrm{A} 15^{22}$ & $\begin{array}{c}\text { Non-technical skills of anaesthetic assistants in the } \\
\text { perioperative period: a literature review }\end{array}$ & 2012 & Reino Unido & $\begin{array}{l}\text { British Journal of } \\
\text { Anaesthesia }\end{array}$ \\
\hline $\mathrm{A} 16^{3}$ & $\begin{array}{c}\text { Improving patient safety in the operating theatre and } \\
\text { perioperative care: obstacles, interventions, and priorities for } \\
\text { accelerating progress }\end{array}$ & 2012 & $\begin{array}{l}\text { Reino Unido, Estados } \\
\text { Unidos }\end{array}$ & $\begin{array}{l}\text { British Journal of } \\
\text { Anaesthesia }\end{array}$ \\
\hline $\mathrm{A} 17^{23}$ & $\begin{array}{c}\text { Implementing an interprofessional patient safety learning } \\
\text { initiative: insights from participants, project leads and } \\
\text { steering committee members }\end{array}$ & 2013 & Canadá & BMJ Quality \& Safety \\
\hline $\mathrm{A} 18^{24}$ & Human factors in resuscitation teaching & 2012 & Reino Unido & Resuscitation \\
\hline A1925 & $\begin{array}{l}\text { How excellent anaesthetists perform in the operating } \\
\text { theatre: a qualitative study on non-technical skills }\end{array}$ & 2013 & Suécia & $\begin{array}{l}\text { British Journal of } \\
\text { Anaesthesia }\end{array}$ \\
\hline $\mathrm{A} 2 \mathrm{O}^{26}$ & Examining patient safety attitudes among urology trainee & 2014 & Reino Unido & BJU International \\
\hline$A 21^{10}$ & $\begin{array}{c}\text { Ensuring patient safety through effective leadership } \\
\text { behavior: a literature review }\end{array}$ & 2010 & Suíça & Safety Science \\
\hline
\end{tabular}


Quadro 1. Continuação.

\begin{tabular}{|c|c|c|c|c|}
\hline Código & Título do artigo & Ano & País & Revista \\
\hline $\mathrm{A} 22^{27}$ & Emotional stability of nurses: impact on patient safety & 2009 & Taiwan & $\begin{array}{l}\text { Journal of Advanced } \\
\text { Nursing }\end{array}$ \\
\hline $\mathrm{A} 23^{28}$ & Does Team Training Work? Principles for Health Care & 2008 & Estados Unidos & $\begin{array}{l}\text { Academic Emergency } \\
\text { Medicine }\end{array}$ \\
\hline $\mathrm{A} 24^{11}$ & $\begin{array}{l}\text { Development and reliability of the explicit professional oral } \\
\text { communication observation tool to quantify the use of non- } \\
\text { technical skills in healthcare }\end{array}$ & 2013 & Holanda & BMJ Quality \& Safety \\
\hline $\mathrm{A} 25^{29}$ & Developing a program, a curriculum, a scenario & 2013 & Estados Unidos & Seminars in Perinatology \\
\hline $\mathrm{A} 26^{30}$ & Cultural safety and the socioethical nurse & 2010 & Nova Zelândia & Nursing Ethics \\
\hline $\mathrm{A} 27^{31}$ & Creating Champions for Health Care Quality and Safety & 2010 & Estados Unidos & $\begin{array}{l}\text { American Journal of } \\
\text { Medical Quality }\end{array}$ \\
\hline $\mathrm{A} 28^{32}$ & $\begin{array}{l}\text { Creating a culture of safety by } \\
\text { coaching clinicians to competence }\end{array}$ & 2013 & Austrália & Nurse Education Today \\
\hline $\mathrm{A} 29^{33}$ & $\begin{array}{l}\text { Simulation for operational readiness in a new freestanding } \\
\text { Emergency Department: strategy and tactics }\end{array}$ & 2016 & Estados Unidos & Simulation in Healthcare \\
\hline $\mathrm{A} 30^{34}$ & $\begin{array}{l}\text { An interprofessional training course in crises and human } \\
\text { factors for perioperative teams }\end{array}$ & 2016 & Reino Unido & $\begin{array}{l}\text { Journal of } \\
\text { Interprofessional Care }\end{array}$ \\
\hline $\mathrm{A} 31^{35}$ & $\begin{array}{l}\text { Strengthening leadership as a catalyst for } \\
\text { enhanced patient safety culture: a repeated } \\
\text { cross-sectional experimental study }\end{array}$ & 2016 & Dinamarca & BMJ Open \\
\hline $\mathrm{A} 32^{36}$ & $\begin{array}{l}\text { Trauma team leaders' non-verbal communication: } \\
\text { video registration during trauma team training }\end{array}$ & 2016 & Suécia & $\begin{array}{l}\text { Scandinavian Journal of } \\
\text { Trauma, Resuscitation and } \\
\text { Emergency Medicine }\end{array}$ \\
\hline $\mathrm{A} 33^{37}$ & $\begin{array}{l}\text { Teamwork education improves trauma team performance } \\
\text { in undergraduate health professional students }\end{array}$ & 2015 & Estados Unidos & $\begin{array}{l}\text { Journal of Educational } \\
\text { Evaluation for Health } \\
\quad \text { Professions }\end{array}$ \\
\hline $\mathrm{A} 34^{38}$ & $\begin{array}{l}\text { Teamwork and clinical error reporting } \\
\text { among nurses in Korean hospitals }\end{array}$ & 2015 & Coreia do Sul & Asian Nursing Research \\
\hline
\end{tabular}

instrumentos de avaliação de habilidades e técnicas para o seu desenvolvimento pelos profissionais de saúde.

Foram identificadas 11 competências: liderança, cultura da segurança, trabalho em equipe, comunicação, advocacia, manejo de risco, competência conceitual, competência funcional, inteligência emocional, tomada de decisão e gestão e planejamento centrado na segurança. Liderança foi citada e analisada em 20 artigos; trabalho em equipe, em 19; comunicação, em 17; e cultura da segurança, em 8. Já o apontamento da advocacia como competência para a segurança do paciente foi apresentado por um artigo apenas.

Os artigos apresentaram os conceitos de competências de modo inovador, considerando as questões relacionadas à segurança do paciente, tais como preocupação com a ocorrência de incidentes, encorajamento para a participação do paciente, minimização de erros, identificação de erros, entre outros (Quadro 2). Verificou-se, ainda, a utilização de técnicas e instrumentos específicos para o desenvolvimento das competências pelos profissionais, apontando para diversos modos de ensinar e aprender nesse contexto.

\section{DISCUSSÃO}

O presente estudo apresenta um número expressivo de artigos que tratam de competências específicas para a segurança do paciente. Todavia, ressalta-se que esse assunto tem uma abordagem recente, visto que as publicações ocorreram a partir de 2008, o que demonstra a necessidade de se olhar para a questão de um modo diferente, isto é, transformar a formação e o desenvolvimento dos profissionais para a prática assistencial e a gestão dos sistemas de saúde ${ }^{15,28}$.

O estudo e o ensino da segurança do paciente são considerados recentes nas instituições assistenciais e formadoras, e a atividade educacional é uma forma de alertar para os riscos dentro do processo assistencial que advêm, em muitos 
Quadro 2. Competências relacionadas à segurança do paciente, definição e instrumentos e técnicas para o seu desenvolvimento.

São Paulo, 2017.

\begin{tabular}{|c|c|c|c|}
\hline Competências & Definição & Instrumentos e Técnicas & Artigos \\
\hline Liderança & $\begin{array}{l}\text { Capacidade de dar direção a um grupo de } \\
\text { pessoas. Tem como objetivos, no contexto } \\
\text { da segurança do paciente, o alcance de } \\
\text { um ambiente acolhedor e livre de culpa e } \\
\text { a construção de uma equipe e organização } \\
\text { centradas na segurança e no alcance de } \\
\text { melhores resultados. É o profissional } \\
\text { quem auxilia na construção da cultura da } \\
\text { segurança na instituição e trabalha para que } \\
\text { ela seja efetiva e afetivamente acolhida por } \\
\text { todos os membros da equipe. }\end{array}$ & 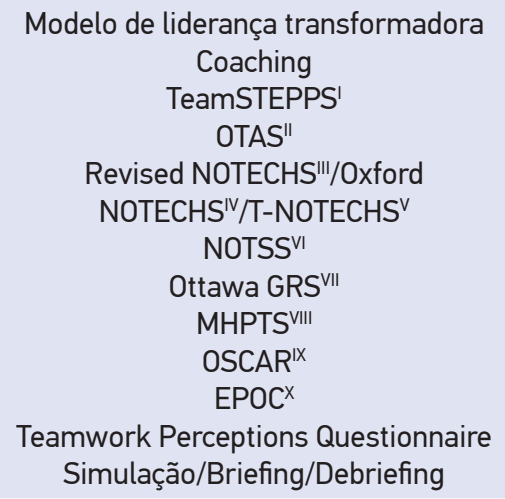 & $\begin{array}{c}\mathrm{A} 1-\mathrm{A} 2-\mathrm{A} 4- \\
\mathrm{A} 6-\mathrm{A} 9-\mathrm{A} 10- \\
\mathrm{A} 12-\mathrm{A} 15-\mathrm{A} 16 \\
-\mathrm{A} 18-\mathrm{A} 20- \\
\mathrm{A} 21-\mathrm{A} 24-\mathrm{A} 25 \\
-\mathrm{A} 28-\mathrm{A} 30- \\
\mathrm{A} 31-\mathrm{A} 32-\mathrm{A} 33 \\
-\mathrm{A} 34\end{array}$ \\
\hline $\begin{array}{l}\text { Cultura da } \\
\text { segurança }\end{array}$ & $\begin{array}{l}\text { Cultura na qual todos os trabalhadores } \\
\text { assumem a responsabilidade pela } \\
\text { segurança, fazendo com que priorizem } \\
\text { a segurança acima de outros interesses. } \\
\text { Oferece encorajamento e recompensas na } \\
\text { identificação, notificação e resolução dos } \\
\text { problemas. Promove, a partir da ocorrência } \\
\text { de incidentes, o aprendizado organizacional. }\end{array}$ & $\begin{array}{c}\text { H-PEPSS } \\
\text { Simulação/Briefing/Debriefing }\end{array}$ & $\begin{array}{c}\mathrm{A} 1-\mathrm{A} 4-\mathrm{A} 5- \\
\mathrm{A} 7-\mathrm{A} 11-\mathrm{A} 12- \\
\mathrm{A} 26-\mathrm{A} 28\end{array}$ \\
\hline Trabalho em equipe & $\begin{array}{l}\text { Atuação conjunta de membros que se apoiam } \\
\text { mutuamente, apresentam comunicação clara } \\
\text { e objetiva e almejam a segurança do paciente. } \\
\text { Para o alcance de boa performance, é } \\
\text { necessário: liderança eficiente, compromisso } \\
\text { e treinamento contínuo. }\end{array}$ & $\begin{array}{c}\text { OSCEXII } \\
\text { H-PEPSS } \\
\text { TeamSTEPPS' } \\
\text { Revised NOTECHS'"I/Oxford } \\
\text { NOTECHS'T-NOTECHS } \\
\text { ANTS }^{\mathrm{XIII}} \\
\text { MHPTS }^{\mathrm{VIII}} \\
\text { OSCAR }^{\mathrm{IX}} \\
\text { EPOC }^{\mathrm{X}} \\
\text { Teamwork Perceptions Questionnaire } \\
\text { Simulação / Briefing / Debriefing }\end{array}$ & $\begin{array}{c}\mathrm{A} 1-\mathrm{A} 2-\mathrm{A} 3-\mathrm{A} 5 \\
-\mathrm{A} 6-\mathrm{A} 7-\mathrm{A} 9- \\
\mathrm{A} 10-\mathrm{A} 15-\mathrm{A} 16 \\
-\mathrm{A} 17-\mathrm{A} 18- \\
\mathrm{A} 20-\mathrm{A} 23-\mathrm{A} 24 \\
-\mathrm{A} 30-\mathrm{A} 32- \\
\text { A33-A34 }\end{array}$ \\
\hline Comunicação & $\begin{array}{l}\text { A comunicação é uma competência chave } \\
\text { para a segurança do paciente, Profissionais } \\
\text { de saúde devem estar aptos para se } \\
\text { comunicarem efetivamente com seus pares } \\
\text { e clientes, para que haja compreensão } \\
\text { mútua, suporte aos relacionamentos } \\
\text { significativos e envolvimento nas decisões } \\
\text { sobre o cuidado. }\end{array}$ & 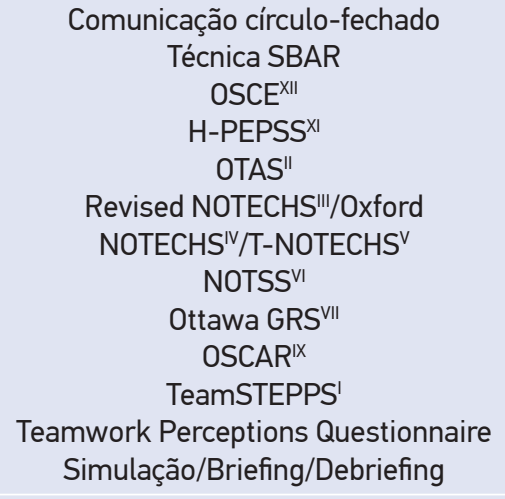 & $\begin{array}{l}\mathrm{A} 1-\mathrm{A} 2-\mathrm{A} 3-\mathrm{A} 4 \\
-\mathrm{A} 5-\mathrm{A} 9-\mathrm{A} 12- \\
\mathrm{A} 13-\mathrm{A} 15-\mathrm{A} 16 \\
-\mathrm{A} 18-\mathrm{A} 24- \\
\mathrm{A} 29-\mathrm{A} 30-\mathrm{A} 32 \\
-\mathrm{A} 33-\mathrm{A} 34\end{array}$ \\
\hline Advocacia & $\begin{array}{l}\text { Ação positiva que resulta em mudança. Seu } \\
\text { exercício favorece a transformação, por meio } \\
\text { da atuação do profissional, da realidade em } \\
\text { que o cuidado acontece. A defesa do paciente } \\
\text { é parte fundamental da advocacia. }\end{array}$ & Simulação/Briefing/Debriefing & A2 \\
\hline Manejo do risco & $\begin{array}{l}\text { Correta identificação dos riscos, com seus } \\
\text { respectivos atores, propostas e execução de } \\
\text { protocolos para prevenção e diminuição de } \\
\text { danos e avaliação de sua eficácia. }\end{array}$ & $\begin{array}{c}\text { H-PEPSS } \\
\text { Simulação/Briefing/Debriefing }\end{array}$ & $\mathrm{A} 5-\mathrm{A} 11-\mathrm{A} 14$ \\
\hline
\end{tabular}


Quadro 2. Continuação.

\begin{tabular}{|c|c|c|c|}
\hline Competências & Definição & Instrumentos e Técnicas & Artigos \\
\hline $\begin{array}{l}\text { Competência } \\
\text { conceitual }\end{array}$ & $\begin{array}{l}\text { Conhecimento profundo e crítico acerca da } \\
\text { temática segurança do paciente para que, } \\
\text { assim, sejam possíveis a real avaliação e o } \\
\text { estabelecimento de propostas de resolução } \\
\text { dos problemas encontrados. }\end{array}$ & Simulação/Briefing/Debriefing & $\begin{array}{c}\mathrm{A} 5-\mathrm{A} 8-\mathrm{A} 10- \\
\mathrm{A} 12-\mathrm{A} 18\end{array}$ \\
\hline $\begin{array}{l}\text { Competência } \\
\text { funcional }\end{array}$ & $\begin{array}{l}\text { Habilidade de transformação do } \\
\text { conhecimento e da teoria em aplicações } \\
\text { práticas, além do efetivo manuseio de } \\
\text { ferramentas que auxiliem as ações. }\end{array}$ & Simulação/Briefing/Debriefing & $\begin{array}{c}\mathrm{A} 8-\mathrm{A} 10-\mathrm{A} 12 \\
-\mathrm{A} 18\end{array}$ \\
\hline $\begin{array}{l}\text { Inteligência } \\
\text { emocional }\end{array}$ & $\begin{array}{l}\text { Capacidade de manter a estabilidade } \\
\text { emocional diante de situações não } \\
\text { planejadas. A estabilidade emocional } \\
\text { tem sido descrita como a tendência de } \\
\text { manter-se confiante e seguro. }\end{array}$ & $\begin{array}{c}\text { Música } \\
\text { Simulação/Briefing/Debriefing }\end{array}$ & $\begin{array}{c}\mathrm{A} 18-\mathrm{A} 19-\mathrm{A} 20 \\
-\mathrm{A} 22\end{array}$ \\
\hline Tomada de decisão & $\begin{array}{l}\text { Ação na qual se avalia determinada situação } \\
\text { com seus desdobramentos e se faz uma } \\
\text { decisão por um caminho determinado. } \\
\text { Na segurança do paciente, essa ação } \\
\text { torna-se mais complexa, pois muitas vezes } \\
\text { ocorrerá a partir de uma situação não } \\
\text { planejada e causadora de estresse. }\end{array}$ & 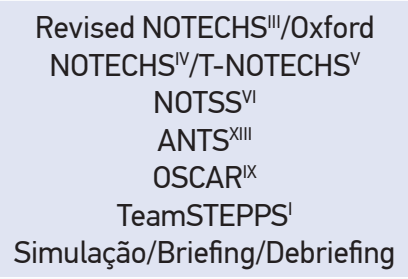 & $\begin{array}{l}\mathrm{A} 9-\mathrm{A} 10-\mathrm{A} 14- \\
\mathrm{A} 15-\mathrm{A} 16-\mathrm{A} 28 \\
-\mathrm{A} 33\end{array}$ \\
\hline $\begin{array}{l}\text { Gestão e } \\
\text { planejamento } \\
\text { centrado na } \\
\text { segurança }\end{array}$ & $\begin{array}{l}\text { Compromisso e ações gerenciais e de } \\
\text { planejamento que têm como prioridade } \\
\text { a segurança do paciente. Permanecem } \\
\text { centralizados e percorrem todas as áreas e } \\
\text { protocolos institucionais. }\end{array}$ & $\begin{array}{c}\text { EPOC }^{1 \mathrm{X}} \\
\text { Simulação/Briefing/Debriefing }\end{array}$ & $\begin{array}{l}\mathrm{A} 2-\mathrm{A} 11-\mathrm{A} 14- \\
\mathrm{A} 19-\mathrm{A} 24-\mathrm{A} 27 \\
-\mathrm{A} 28\end{array}$ \\
\hline
\end{tabular}

Team Strategies and Tools to Enhance Performance and Patient Safety; "Observational Teamwork Assessment for Surgery; "'Revised Non-technical Skills; ' $\mathrm{V}$-xford Non-technical Skills; ${ }^{\vee}$ Trauma Non-technical Skills; "Non-technical Skills for Surgeons; "IIIttawa Crisis Resource Management Global Rating Scale; "il'Mayo High Performance Teamwork Scale; 'Observational Skill-based Clinical Assessment Tool for Resuscitation; ${ }^{\mathrm{X}}$ Explicit Professional Oral Communication; ${ }^{\mathrm{x}}$ Health Professional Education in Patient Safety Survey; ${ }^{\mathrm{XI}}$ Objective Structured Clinical Exam; ${ }^{\mathrm{XI}}$ Anesthesiologists Non-technical Skills.

casos, das falhas operacionais do sistema de atendimento. Portanto, é esperado que esses estudos estejam vinculados aos Estados Unidos, ao Reino Unido, ao Canadá e à Austrália, que foram precursores nas pesquisas relacionadas à temática, propiciaram o entendimento dos fatores causais e culturais e introduziram iniciativas abrangentes em seus territórios, de modo a modificar a cultura dentro das organizações ${ }^{7,14,32,34}$.

Embora estudos brasileiros relacionados a erros e eventos adversos dentro do sistema de saúde não sejam escassos, o estudo das competências ainda é um desafio para os pesquisadores no cenário nacional. Espera-se um maior amadurecimento nas organizações de assistência e de formação, induzido pela necessidade de se implantar o Programa Nacional de Segurança do Paciente, lançado pelo Ministério da Saúde, em 2003, que objetiva monitorar e prevenir, em hospitais e outras unidades de saúde, os incidentes causadores de danos na assistência ao usuário ${ }^{39}$.

No estudo em questão $0^{39}$, as competências mais frequentes foram liderança, trabalho em equipe e comunicação. Estudos sobre liderança não são incomuns na literatura; todavia, a liderança voltada para a segurança do paciente destaca um líder que propicia um ambiente acolhedor e livre de culpa, possibilitando o crescimento da equipe nas questões que dizem respeito à segurança do paciente, transformando a realidade vigente relacionada a punições e advertências diante de erros em um ambiente de aprendizado ${ }^{13,26,35,36,38}$.

O trabalho em equipe é essencial para a segurança do paciente e sua importância tende a aumentar devido a fatores como maior complexidade das doenças, crescimento das especializações no atendimento, elevação de comorbidades, escassez de força de trabalho, entre outros. Os trabalhos em saúde envolvem muitos profissionais e precisam ser bem coordenados, devendo haver boa comunicação entre eles em todos os momentos. Assim, o trabalho em equipe destaca-se como uma competência fundamental ${ }^{6,14,34,37}$. O cenário cirúrgico é um excelente exemplo dessa importância, e a implantação do protocolo de cirurgia segura é uma ferramenta que propicia a participação dos profissionais no sentido de evitar erros e eventos adversos relacionados aos procedimentos ${ }^{1}$. 
Contudo, para que se faça um trabalho em equipe adequado, que significa desenvolver um trabalho coletivo, com diversas intervenções técnicas, interação de profissionais de diferentes áreas e construído por meio de uma relação mutual, é necessário observar uma de suas premissas principais: a qualidade da comunicação entre os integrantes. Sabe-se que o fator causal mais frequente para a ocorrência de erros e eventos adversos dentro do sistema de saúde é a comunicação inadequada ${ }^{8,11,36,37}$.

Por essa razão, inúmeros estudos têm sido conduzidos no sentido de estabelecer uma comunicação efetiva entre os membros da equipe, por meio de um processo estruturado e honesto entre os profissionais de saúde e os pacientes, principalmente após estes terem sofrido danos ${ }^{8,11,17,38}$.

Verifica-se, também, que surgem novas competências relacionadas à segurança do paciente, como a advocacia, que tem foco na defesa do paciente, tornando-o prioritário dentro do serviço em contraponto às questões relacionadas à estrutura ou à organização. Trata-se de uma preocupação com o paciente em relação a seu tratamento e sua necessidade de cuidados, à sua compreensão diante dos consentimentos informados e ao estímulo para o esclarecimento de dúvidas quanto às suas necessidades assistenciais. Tal competência implica em saber ouvir o paciente, saber negociar, conhecer a equipe de trabalho, ter conhecimentos apurados sobre o processo administrativo e as ferramentas de melhoria da qualidade ${ }^{14,15,17}$.

O presente estudo ainda indica o desenvolvimento das competências com instrumentos e técnicas diferenciados, o que permite supor que o ensino e a aprendizagem realizados de forma tradicional, dentro de um paradigma positivista, disciplinarmente organizado, não cabem no contexto da segurança do paciente. Dessa forma, educadores, gestores e professores, além de estarem continuamente preparados em seus conhecimentos teóricos, devem utilizar metodologias educativas inovadoras, que permitam aos profissionais e estudantes transformar informações em conhecimentos significativos e aplicáveis ${ }^{8,17,29,33,36}$.

A limitação deste estudo centra-se na utilização de apenas uma base de dados para a busca de artigos. Porém, o material encontrado e a análise feita permitem identificar competências importantes para a gestão da segurança do paciente, bem como indicam a possibilidade de se realizar novos estudos que aprimorem o seu desenvolvimento.

\section{CONCLUSÃO}

A identificação das competências relacionadas à segurança do paciente possibilitou defini-las no contexto da gestão e, principalmente, verificar estratégias para o seu desenvolvimento. Estudos subsequentes podem aplicar essas estratégias e testar sua evidência. Isso possibilitará aos gestores das instituições planejar a implementação da cultura de segurança de uma maneira mais efetiva e concreta, trazendo inúmeros benefícios às organizações e a seus clientes.

\section{REFERÊNCIAS}

1. World Health Organization. Medication without harm: WHO's Third Global Patient Safety Challenge [Internet]. Geneva: World Health Organization, 2017 [cited 2017 July 8]. Available from: http://who. int/patientsafety/medication-safety/en/

2. Naik VN, Brien SE. Review article: simulation: a means to address and improve patient safety. Can J Anaesth. 2013;60(2):192-200.

3. Sevdalis N, Hull L, Birnbach DJ. Improving patient safety in the operating theatre and perioperative care: obstacles, interventions, and priorities for accelerating progress. Br J Anaesth. 2012;109:i3-16.

4. Landrigan CP, Parry GJ, Bones CB, Hackbarth AD, Phil M, Goldmann DA, et al. Temporal trends in rates of patient harm resulting from medical care. N Engl J Med. 2010;363:2124-34.

5. Fleury MTL. Construindo o conceito de competência. Rev Adm Contemp. 2001:183-96.
6. Ginsburg L, Castel E, Tregunno D, Norton PG. The H-PEPSS: an instrument to measure health professionals' perceptions of patient safety competence at entry into practice. BMJ Qual Saf. 2012;21:676-84.

7. Ginsburg L, Tregunno D, Norton PG. Self-reported patient safety competence among new graduates in medicine, nursing and pharmacy. BMJ Qual Saf. 2013;22:147-54.

8. Cushing AM, Ker JS, Kinnersley P, McKeown P, Silverman J, Patterson $\mathrm{J}$, et al. Patient safety and communication: a new assessment for doctors trained in countries where language differs from that of the host country: results of a pilot using a domain-based assessment. Patient Educ Couns. 2014;95(3):332-9.

9. Dempsey J. Nurses values, attitudes and behavior related to falls prevention. J Clin Nurs. 2009;18(6):838-48. 
10. Kunzle B, Kolbe M, Grote G. Ensuring patient safety through effective leadership behavior: a literature review. Saf Sci. 2010;48(1):1-17.

11. Kemper PF, Noord IV, Bruijne M, Knol DL, Wagner C, Dyck CV. Development and reliability of the explicit professional oral communication observation tool to quantify the use of non-technical skills in healthcare. BMJ Qual Saf. 2013;22(7):586-95.

12. Mendes KDS, Silveira RCCP, Galvão CM. Revisão integrativa: método de pesquisa para a incorporação de evidências na saúde e na enfermagem. Texto Contexto Enferm. 2008;17(4):758-764.

13. Siassakos D, Fox R, Bristowe K, Angouri J, Hambly H, Robson L, et al. What's makes maternity teams effective and safe? Lessons from a series of research on teamwork, leadership and team training. Acta Obstet Gynecol Scand. 2013;92(11):1239-43.

14. Aggarwal R, Mytton OT, Derbrew M, Hananel D, Heydenburg $\mathrm{M}$, Issenberg B, et al. Training and simulation for patient safety. Qual Saf Health Care. 2010;19(Suppl 2):i34-43.

15. Wagner DP, Hoppe RB, Lee CP. The Patient Safety OSCE for PGY-1 Residents: A Centralized response to the challenge of culture change. Teach Learn Med. 2009;21(1):8-14.

16. Mcfadden KL, Henagan SC, Gowen CR. The patient safety chain: Transformational leadership's effect on patient safety culture, initiatives, and outcomes. J Operations Manag. 2009;27(5):390-404.

17. Sanfey H, McDowell C, Meier AH, Dunnington GL. Team training for surgical trainee. Surg (Edinb). 2011;9(Suppl 1):S32-4.

18. Weeks KW, Hutton BM, Young S, Coben D, Clochesy JM, Pontin D. Safety in Numbers 2: Competency modelling and diagnostic error assessment in medication dosage calculation problem-solving. Nurse Educ Pract. 2013;13(2)e23-32.

19. Azuara-Blanco A, Reddy A, Wilkinson G, Flin R. Safe eye surgery: non-technical aspects. Eye (Lond). 2011;25(9):1109-11.

20. Russell TR. Quality and safety initiatives in the future practice of surgery: meeting patient demands for enhanced professionalism. Surg Today. 2009;39(9):739-45.

21. Keats JP. Patient safety in the obstetric and gynecologic office setting. Obstet Gynecol Clin North Am. 2013;40(4):611-23.

22. Rutherford JS, Flin R, Mitchell L. Non-technical skills of anaesthetic assistants in the perioperative period: a literature review. $\mathrm{Br} \mathrm{J}$ Anaesth. 2012;109(1):27-31.

23. Jeffs L, Abramovich IA, Hayes C, Smith O, Tregunno D, Chan WH, et al. Implementing an interprofessional patient safety learning initiative: insights from participants, project leads and steering committee members. BMJ Qual Saf. 2013;22(11):923-30.

24. Norris EM, Lockey AS. Human factors in resuscitation teaching. Resuscitation. 2012;83(4):423-7.
25. Larsson J, Holmström IK. How excellent anaesthetists perform in the operating theatre: a qualitative study on non-technical skills. $\mathrm{Br}$ J Anaesth. 2013;110(1):115-21.

26. Geraghty A, Reid S, Mcllhenny C. Examining patient safety attitudes among urology trainee. BJU Int. 2014;113(1):167-75.

27. Teng $\mathrm{Cl}$, Chang SS, Hsu KH. Emotional stability of nurses: impact on patient safety. J Adv Nurs. 2009;65(10):2088-96.

28. Salas E, Dias-Granados D, Weaver SJ, King H. Does team training work? Principles for health care. Acad Emerg Med. 2008;15(11):1002-9.

29. Birsner ML, Satin AJ. Developing a program, a curriculum, a scenario. Semin Perinatol. 2013;37(3):175-8.

30. Woods M. Cultural safety and the socioethical nurse. Nurs Ethics. 2010 Nov; 17(6):715-25.

31. Holland R, Meyers D, Hildebrand C, Bridges AJ, Roach MA, Vogelman B. Creating champions for health care quality and safety. Am J Med Qual. 2010 Mar-Apr;25(2):102-8.

32. Duff B. Creating a culture of safety by coaching clinicians to competence. Nurse Educ Today. 2013;33(10):1108-11.

33. Kerner Jr. RL, Gallo K, Cassara M, D'Angelo J, Egan A, Simmons JG. Simulation for operational readiness in a new freestanding Emergency Department: strategy and tactics. Simul Healthc. 2016;11(5):345-56.

34. Stephens T, Hunningher A, Mills H, Freeth D. An interprofessional training course in crises and human factors for perioperative teams. J Interprof Care. 2016;30(5):685-8.

35. Kristensen S, Christensen KB, Jaquet A, Beck CM, Sabroe S, Bartels $\mathrm{P}$, et al. Strengthening leadership as a catalyst for enhanced patient safety culture: a repeated cross-sectional experimental study. BMJ Open. 2016;6(5):e010180.

36. Härgestam M, Hultin M, Brulin C, Jacobsson M. Trauma team leaders' non-verbal communication: video registration during trauma team training. Scand J Trauma Resusc Emerg Med. 2016;24:37.

37. Baker VO, Cuzzola R, Knox C, Liotta C, Cornfield CS, Tarkowski RD, et al. Teamwork education improves trauma team performance in undergraduate health professional students. J Educ Eval Health Prof. 2015;12:36.

38. Hwang JI, Ahn J. Teamwork and clinical error reporting among nurses in Korean hospitals. Asian Nurs Res (Korean Soc Nurs Sci). 2015;9(1):14-20.

39. Brasil. Ministério da Saúde. Portaria n 529, de 1 de abril de 2013. Institui o Programa Nacional de Segurança do Paciente (PNSP). Diário Oficial da União [Internet]. 2013 [cited on 2015 Aug 14];Seção1:43-4. Available from: http://bvsms.saude.gov.br/bvs/saudelegis/gm/2013/ prt0529_01_04_2013.html 